Orthopäde $2011 \cdot 40: 584$

DOI 10.1007/s00132-011-1816-4

Online publiziert: 17. Juni 2011

(c) Springer-Verlag 2011

\title{
V. Ewerbeck
}

Abteilung Orthopädie, Stiftung Orthopädische Universitätsklinik, Heidelberg

\section{Lernen aus Fehlern - auch in der Wissenschaft ein Standardprozess}

C. Schulz et al. weisen in der Arbeit zum Thema „Perkutane CT-gesteuerte Destruktion vs. mikrochirurgische Resektion lumbaler Juxtafacettenzysten“ nach, dass die perkutane, CT-gesteuerte Destruktion lumbaler Juxtafacettenzysten (minimalinvasiv!) hinsichtlich der dauerhaften Erfolgsquote gegenüber der mikrochirurgischen Vorgehensweise kein konkurrenzfähiges Verfahren ist.

\section{() Auch negative Ergebnisse führen zu einem Lerneffekt}

Und schließlich zeigten C. von SchulzePellengahr et al. in ihren Untersuchungen zum Thema „Primärstabilität zementfreier Hüftpfannen bei Osteoporose“, dass reduzierte Knochendichte nicht zu einer reduzierten Primärstabilität bestimmter zementfreier Hüftpfannen unter Zugrundelegung der Mikrobewegungen als Messparameter führt.

Die alte Erkenntnis, dass nicht nur positive, sondern auch negative Ergebnisse zu Lerneffekten führen, erfährt ein weiteres Mal ihre Bestätigung. Besonders gut lernen wir aus Fehlern.

Abseits davon präsentieren wir Ihnen zur Beurteilung eine neue Klassifikation zur Entscheidungsfindung zur Therapie der „Pyogenen Spondylodiszitis der Brust- und Lendenwirbelsäule“ (M. Akbar et al.) sowie zwei, wie wir glauben, interessante kasuistische Mitteilungen.

Wie stets bemüht sich Der Orthopäde darum, dass sein Inhalt möglichst nahe an der klinischen Realität seiner Leser angesiedelt wird. Ich hoffe, dass uns das auch dieses Mal gelungen ist und bleibe mit besten kollegialen Grüßen
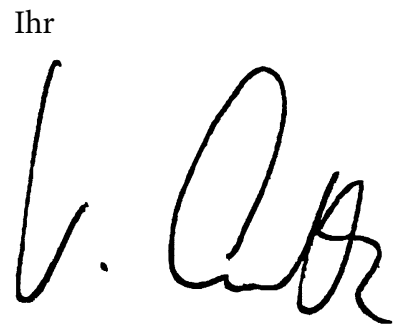

Prof. Dr. med. V. Ewerbeck

\section{Korrespondenzadresse \\ Prof. Dr. V. Ewerbeck}

Abteilung Orthopädie,

Stiftung Orthopädische Universitätsklinik

Schlierbacher Landstraße 200a,

69118 Heidelberg

Volker.Ewerbeck@med.uni-heidelberg.de 